\title{
Use of Non-Echo-Planar Diffusion-Weighted MR Imaging for the Detection of Cholesteatomas in High-Risk Tympanic Retraction Pockets
}

\author{
A. Alvo, C. Garrido, Á. Salas, G. Miranda, C.E. Stott, and P.H. Delano
}

\begin{abstract}
BACKGROUND AND PURPOSE: Non-echo-planar DWI MR imaging (including the HASTE sequence) has been shown to be highly sensitive and specific for large cholesteatomas. The purpose of this study was to determine the diagnostic accuracy of HASTE DWI for the detection of incipient cholesteatoma in high-risk retraction pockets.
\end{abstract}

MATERIALS AND METHODS: This was a prospective study of 16 patients who underwent MR imaging with HASTE DWI before surgery. Surgeons were not informed of the results, and intraoperative findings were compared against the radiologic diagnosis. Sensitivity, specificity, and positive and negative predictive values were calculated.

RESULTS: Among the 16 retraction pockets, 10 cholesteatomas were diagnosed intraoperatively (62.5\%). HASTE showed $90 \%$ sensitivity, $100 \%$ specificity, $100 \%$ positive predictive value, and $85.7 \%$ negative predictive value in this group of patients. We found only $1 \mathrm{false}-$ negative finding in an infected cholesteatoma.

CONCLUSIONS: We demonstrate a high correlation between HASTE and surgical findings, suggesting that this technique could be useful for the early detection of primary acquired cholesteatomas arising from retraction pockets and could help to avoid unnecessary surgery.

ABBREVIATION: EP $=$ echo-planar

M iddle ear cholesteatomas are benign but locally aggressive nonneoplastic lesions composed of a keratinizing stratified squamous epithelial matrix, an inflammatory perimatrix, and desquamated keratin content. ${ }^{1}$ Pathophysiologically, cholesteatomas are divided into congenital (epithelium trapped within the middle ear during fetal development) and acquired. Acquired cholesteatomas are further divided into primary (arising from a tympanic membrane retraction) and secondary (epithelium reaching the middle ear through a tympanic perforation, fracture, or iatrogenic procedure). ${ }^{2,3}$ Cholesteatomas progressively erode the bony structures surrounding the middle ear (ossicles, facial nerve canal, bony labyrinth, and skull base), predisposing to a wide range of complications, including potentially severe infec-

Received December 26, 2013; accepted after revision February 11, 2014.

From the Departments of Otorhinolaryngology (A.A., C.E.S., P.H.D.) and Radiology (C.G., A.S., G.M.), Hospital Clínico Universidad de Chile, Santiago, Chile.

This work was financed with funds from the Sociedad Chilena de Otorrinolaringología-Medicina y Cirugía de Cabeza y Cuello.

Please address correspondence to Andrés Alvo, MD, Servicio de Otorrinolaringología, Hospital Clínico Universidad de Chile, Santos Dumont 999, Independencia. Santiago, Chile, 8380456; e-mail: andresalvo@gmail.com; and Paul H. Delano, MD, Servicio de Otorrinolaringología, Hospital Clínico Universidad de Chile, Instituto de Ciencias Biomédicas. Facultad de Medicina, Universidad de Chile, Santos Dumont 999, Independencia. Santiago, Chile, 8380456; e-mail: phdelano@gmail.com

http://dx.doi.org/10.3174/ajnr.A3952 tions such as meningitis and intracranial abscesses. ${ }^{4}$ Surgery is the only known curative treatment and should be performed early because less destruction allows more conservative and hearingpreserving procedures with a reduced risk of complications.

Tympanic retraction pockets are invaginations of the tympanic membrane into the middle ear cleft caused by Eustachian tube dysfunction, which interferes with proper middle ear ventilation. ${ }^{5}$ The diagnosis of a "dangerous" or high-risk retraction pocket is proposed when the bottom of the pocket becomes hidden to the otomicroscope and/or starts retaining skin, because this may lead to primary acquired cholesteatoma. ${ }^{1}$ Given that there is a continuum from tympanic retraction pockets to small cholesteatomas, the distinction between retraction pockets that have already developed a cholesteatoma and those that have not remains a problem in otologic surgery because conventional clinical and radiologic methods are often insufficient. In addition, dangerous retraction pockets and small cholesteatomas share similar clinical signs and symptoms, and it is often impossible to differentiate them via otomicroscopy. A substantial number of such patients undergo surgical procedures such as mastoidectomies or atticotomies, but only a subset actually have cholesteatomas.

$\mathrm{CT}$ is the most widely used imaging technique for the detection of a middle ear mass and assessing tympanomastoid anatomy 
and the extent of bone erosion. ${ }^{6}$ However, it is nonspecific for cholesteatoma and relies on indirect signs for its diagnosis. ${ }^{7,8}$ More recently, MR imaging techniques have been used to differentiate cholesteatoma from other middle ear masses, especially T1-weighted delayed postcontrast imaging and DWI. ${ }^{9,10}$

DWI techniques are based on the restriction of movement of water molecules, such as that caused by keratin-filled cholesteatomas, producing a hyperintense signal. ${ }^{11}$ Conventional EPI has been displaced by non-EPI techniques when the temporal bone is the focus, because these sequences have fewer artifacts and thinner sections, allowing the detection of cholesteatomas as small as $2 \mathrm{~mm} .{ }^{12}$ Several studies of non-echo-planar (EP) DWI have provided excellent sensitivity and specificity for the detection of cholesteatomas. Currently, MR imaging is suggested when the diagnosis of cholesteatoma cannot be established by other means, often in the setting of congenital and residual/recurrent disease. ${ }^{10}$

The purpose of this study was to evaluate the sensitivity and specificity of non-EP DWI for the detection of cholesteatomas in skin-retaining and/or otomicroscopically inaccessible tympanic retraction pockets.

\section{MATERIALS AND METHODS Research Design}

A prospective study was conducted at the Department of Otorhinolaryngology in our institution. HASTE non-EP DWI MR imaging was performed within 2 months before surgery, in addition to standard examinations, including audiometry and temporal bone CT. HASTE images were blindly interpreted by an experienced neuroradiologist, and radiologic diagnosis of cholesteatoma was considered when a marked hyperintensity in comparison with brain tissue was noted on DWI. After all patients had been recruited, a second neuroradiologist revised all HASTE images to evaluate the interobserver agreement of the test.

Patients with tympanic membrane retraction pockets in which a cholesteatoma could not be ruled out or confirmed were included. Exclusion criteria included previously operated ears, clinically evident cholesteatomas, contraindications for MR imaging, and refusal by the patient. Staff otologists with at least 5 years of experience in middle ear surgery performed all operations. Surgeons were blind to the results of the MR imaging and independently determined the presence or absence of cholesteatoma in the middle ear. The study was approved by the ethics committee of our hospital (approval No. 68, 2011).

\section{Patients}

Sixteen consecutive patients clinically diagnosed with tympanic retraction pockets were recruited and evaluated by an expert otology committee. When a high-risk pocket was scheduled for surgery, the patient was considered a possible candidate and offered an informed consent form. Anonymity was preserved throughout the study.

\section{Imaging Technique}

All MRI was performed by using a $1.5 \mathrm{~T}$ superconducting MR imaging scanner (Magnetom Symphony Maestro Class; Siemens, Erlangen, Germany) with 20-mT/m maximum amplitude gradients. All images were acquired by using a 4-channel circularly

\begin{tabular}{ccccccc}
\multicolumn{7}{c}{ Patient characteristics and MRI and surgical findings } \\
\hline $\begin{array}{c}\text { Patient } \\
\text { No. }\end{array}$ & Sex & $\begin{array}{c}\text { Age } \\
\text { (yr) }\end{array}$ & Side & HASTE & $\begin{array}{c}\text { Size } \\
\text { (mm) }\end{array}$ & Surgery \\
\hline 1 & F & 27 & L & + & 3 & + \\
2 & M & 21 & R & - & 0 & - \\
3 & F & 39 & R & - & 0 & - \\
4 & F & 35 & L & - & 0 & - \\
5 & M & 29 & R & + & 13 & + \\
6 & M & 43 & R & + & 6 & + \\
7 & F & 39 & L & + & 5 & + \\
8 & F & 55 & L & + & 6 & + \\
9 & M & 49 & R & - & 0 & + \\
10 & F & 42 & R & + & 5 & + \\
11 & F & 60 & L & + & 10 & + \\
12 & M & 9 & L & + & 15 & + \\
13 & F & 58 & R & - & 0 & - \\
14 & F & 48 & L & + & 4 & + \\
15 & M & 24 & L & - & 0 & - \\
16 & F & 43 & R & - & 0 & - \\
\hline
\end{tabular}

Note:- $\mathrm{L}$ indicates left; $\mathrm{R}$, right; + , positive finding; - , negative finding.

polarized head-array coil. To obtain the imaging planes, we performed the following protocol: sagittal T1 turbo inversion recovery magnitude dark fluid sequence (TR, $1710 \mathrm{~ms}$; TE, $12 \mathrm{~ms}$; TI, $860 \mathrm{~ms}$; flip angle, $150^{\circ}$; matrix, $202 \times 256$; FOV, $219 \times 250$; section thickness, $5 \mathrm{~mm}$; NEX, 1; twenty sections; acquisition time, 1 minute 44 seconds); axial and coronal T2 TSE imaging with a restore pulse (TR, $5350 \mathrm{~ms}$; TE, $110 \mathrm{~ms}$; flip angle, $180^{\circ}$; matrix, $220 \times 384$; FOV, $172 \times 180$; section thickness, $3 \mathrm{~mm}$; NEX, 2; twenty sections; acquisition time, 2 minutes 26 seconds); axial T2 CISS sequence, -64 partitions in $1 \mathrm{slab}, 0.8 \mathrm{~mm}$ in the axial plane (TR, $10.86 \mathrm{~ms}$; TE, 5.43 ms; flip angle, $70^{\circ}$; matrix, $208 \times 256$; FOV, $146 \times 180$; NEX, 1; acquisition time, 4 minutes 16 seconds); and axial and coronal HASTE sequences (TR, $2700 \mathrm{~ms}$; TE, $132 \mathrm{~ms}$; flip angle, $180^{\circ}$; turbo factor, 256; matrix, $256 \times 256$; FOV, $200 \times 200$; section thickness, 3 $\mathrm{mm}$; intersection gap, $0 \mathrm{~mm}$; NEX, 4; b factor, $800 \mathrm{~s} / \mathrm{mm}^{2}$; $14 \mathrm{sec}-$ tions; acquisition time, 2 minutes 33 seconds). Unless dural involvement secondary to middle fossa bone erosion was suspected, all sequences were performed without intravenous injection of gadolinium-based contrast media.

\section{Statistical Analysis}

Patients were dichotomically classified as positive or negative for cholesteatoma. Dubious cases were considered as positive, given that a cholesteatoma could not be ruled out. Data were tabulated in a spreadsheet application (Excel for Mac 2011; Microsoft, Bothell, Washington). Diagnostic tests were evaluated by comparing HASTE results against the intraoperative findings. Statistical measures, including sensitivity, specificity, positive predictive value, and negative predictive value, were calculated. Interobserver agreement was calculated by using the $\kappa$ coefficient.

\section{RESULTS}

Sixteen patients (6 males [37.5\%] and 10 females [62.5\%]) with ages ranging from 9 to 60 years (mean, $38.8 \pm 14.1$ years) were included during 2 years (November 2011 to November 2013). There was no difference in the laterality of the disease ( 8 left- and 8 right-sided).

Intraoperatively, we found cholesteatomas in 10 of 16 retraction pockets $(62.5 \%)$. Using HASTE resulted in 9 true-positives, 

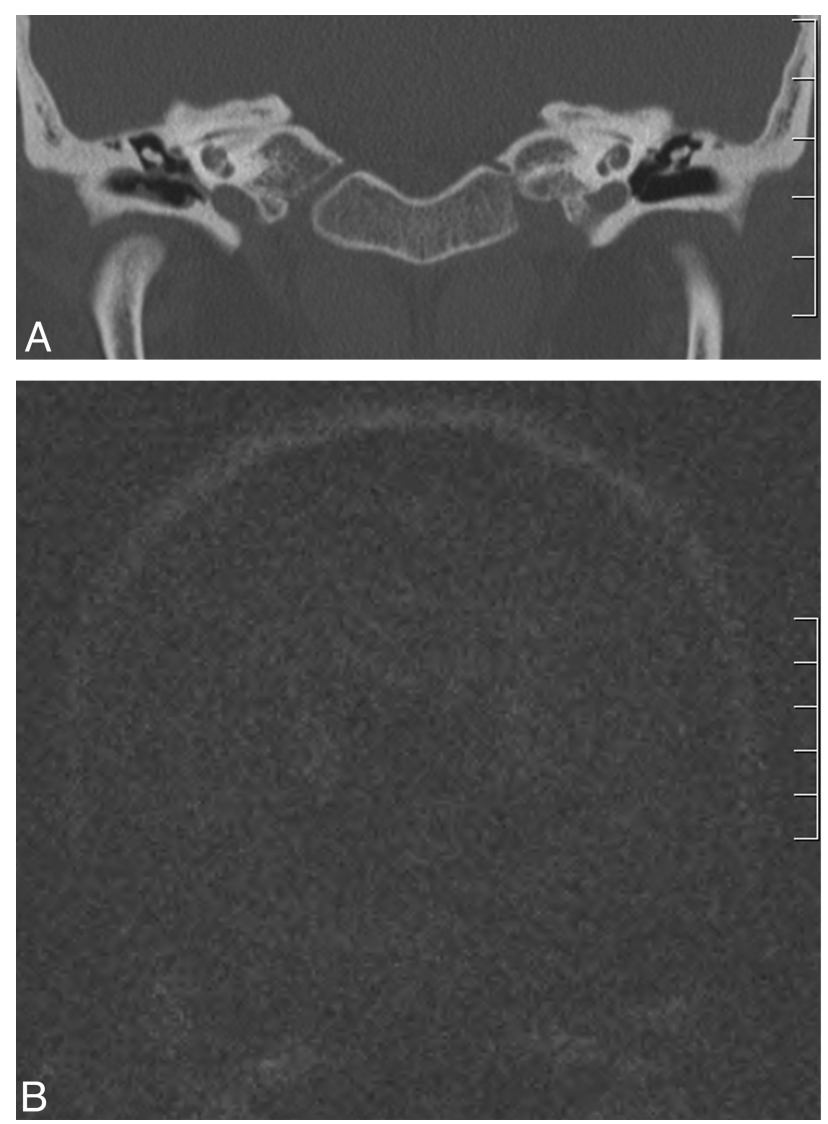

FIG 1. Case 3. A 39-year-old woman with no prior otologic diagnosis. $A$, Coronal $\mathrm{CT}$ scan shows right tympanic membrane thickening with occupation of Prussak space. B, Coronal HASTE shows no hyperintensity. In surgery, a granulomatous mass was identified without evidence of cholesteatoma (not shown).

zero false-positives, 1 false-negative, and 6 true-negatives (Table). Radiologically, the size of the cholesteatomas ranged between 3 and $15 \mathrm{~mm}$ (mean, $7.4 \mathrm{~mm}$ ). Calculated statistical measures for HASTE with their respective $95 \%$ confidence intervals were as follows: $90 \%$ sensitivity $(55.5 \%-99.8 \%), \quad 100 \%$ specificity (54.1\%-100\%), 100\% positive predictive value $(66.4 \%-100 \%)$, and $85.7 \%$ negative predictive value $(42.1 \%-99.6 \%)$.

Further analysis included a second neuroradiologist to evaluate the interobserver agreement; the calculated $\kappa$ coefficient was 0.875 $(0.640-1.000)$. There was an additional false-negative in this retrospective analysis, which had been classed as "faintly positive" (a small, weak, hyperintense signal, different from the surrounding tissues) by the first neuroradiologist. Sample images that are representative of different clinical situations are shown in Figs 1-3.

\section{DISCUSSION}

Our results show that HASTE can predict the presence of cholesteatomas in cases of retraction pockets that retain skin or cannot be fully evaluated by direct otomicroscopy and in which the diagnosis is uncertain. The presence of keratin in the cholesteatoma leads to a restrictive pattern with characteristic hyperintensity on DWI, and the use of non-EPI enabled the avoidance of susceptibility artifacts at the skull base, which can be mistaken for or hide a cholesteatoma. ${ }^{12}$

Common diagnostic techniques for cholesteatoma, including
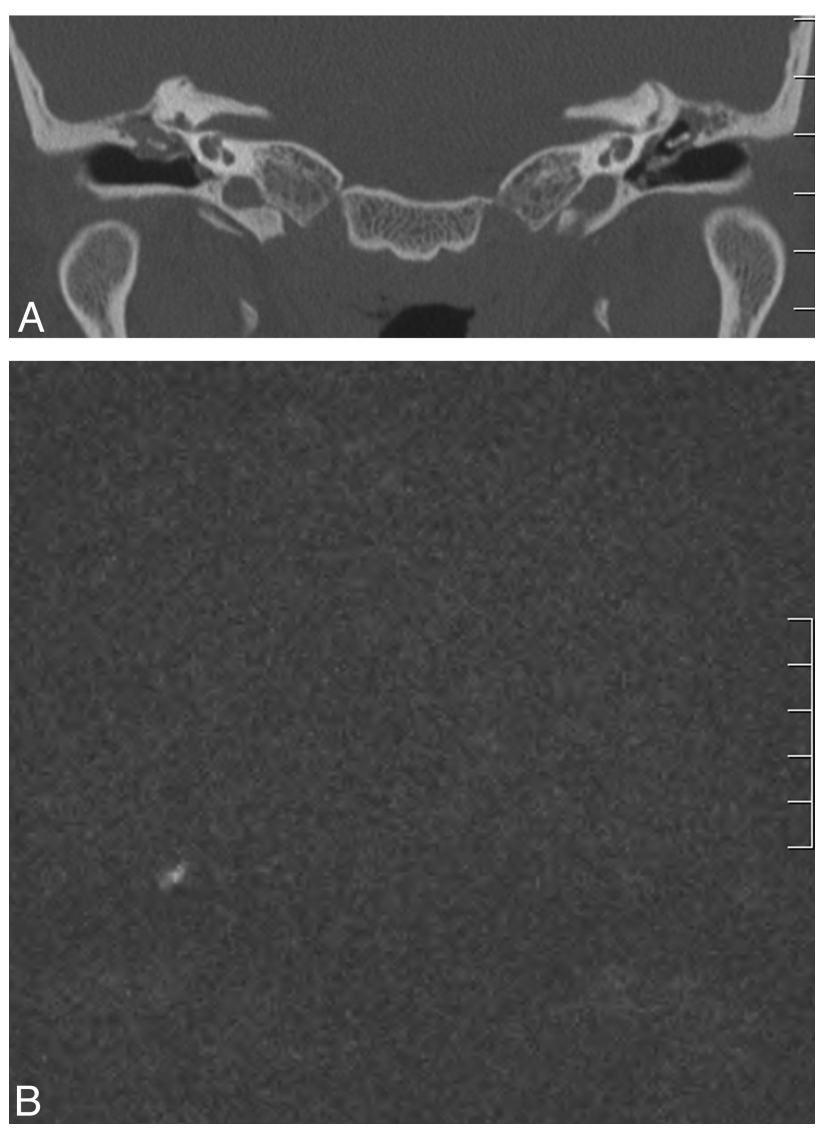

FIG 2. Case 5. A 29-year-old man with a history of chronic bilateral otitis media with effusion with a tympanic ventilation T-tube on the left side. A, Coronal CT scan shows bilateral tympanic thickening and middle ear opacities, with erosion of the scutum; a T-tube can be observed on the left side. $B$, Coronal HASTE shows a 13-mm rightsided hyperintensity in relation to the epitympanum. The cholesteatoma was found intraoperatively (not shown).

otomicroscopy and CT, are useful but, in many cases, insufficient. Ganaha et $\mathrm{al}^{13}$ reported sensitivities, specificities, positive predictive values, and negative predictive values of $77.9 \%, 92.8 \%$, $97.8 \%$, and $50.0 \%$ for otoscopy and $71.1 \%, 78.5 \%, 93.3 \%$, and $39.2 \%$ for CT, respectively. Although the diagnosis of large cholesteatomas can be adequately accomplished by these methods, detection rates as low as $50 \%-60 \%$ have been described in difficult cases such as residual, recurrent, and congenital cholesteatomas. ${ }^{13}$ In addition, the low negative predictive value for CT and otomicroscopy suggests that in questionable cases, like differentiating a small cholesteatoma and a noncholesteatomatous tympanic retraction pocket, further diagnostic tools are needed to rule out the presence of a middle ear cholesteatoma. For these reasons, MR imaging techniques have been proposed for the evaluation of middle ear disease.

\section{Conventional MR Imaging Sequences for the Diagnosis of Cholesteatoma}

Cholesteatomas can be difficult to detect and/or appear as nonspecific lesions on standard MR images, ${ }^{11,14,15}$ but keratin shows a restrictive pattern on DWI. Conventional EP DWI, however, has not yielded sufficiently precise results, especially for cholesteatomas smaller than $5 \mathrm{~mm}$. Jindal et $\mathrm{al}^{11}$ reported a sensitivity of $83 \%$ 

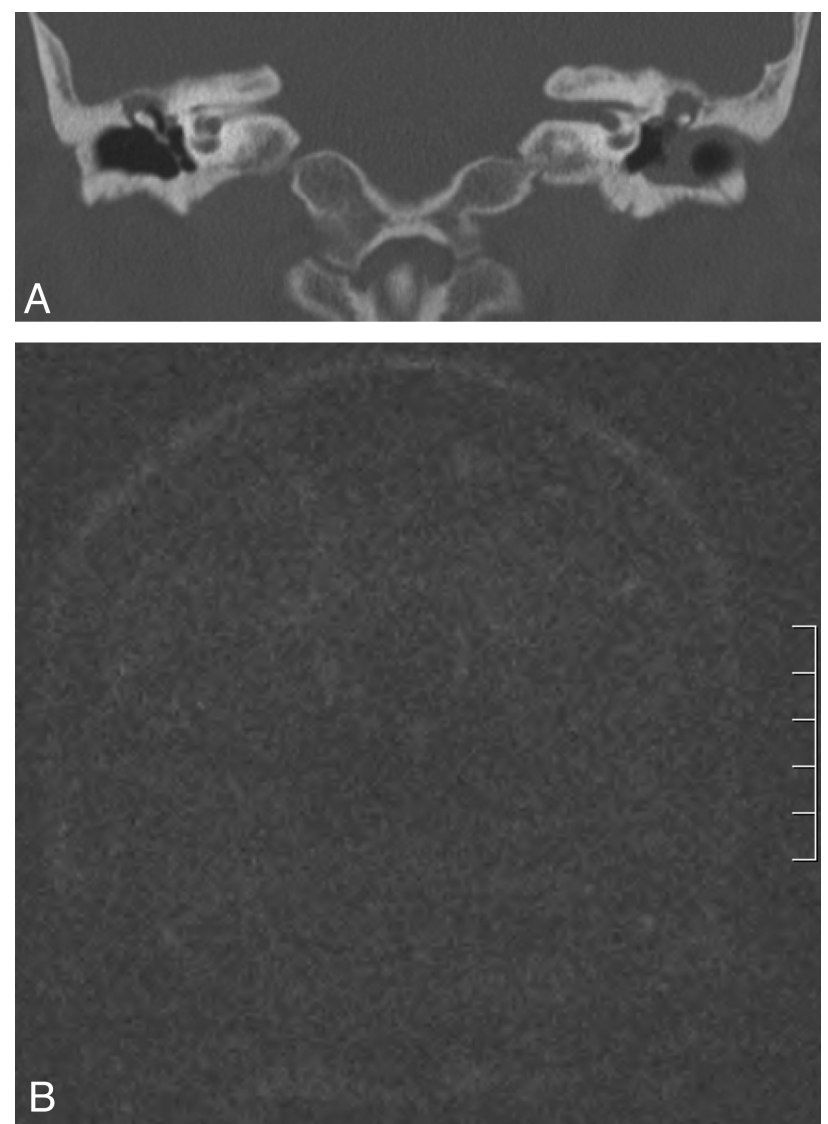

FIG 3. Case 13. A 58-year-old woman with bilateral chronic ear disease (right tympanic retraction pocket and left external ear canal stenosis). A, Coronal CT shows a right-sided tympanic retraction pocket with partial thickening of the tympanic membrane and erosion of the scutum, with an epitympanic middle ear mass. On the left side, there is external ear canal occupation by a soft-tissue attenuation mass, with epitympanic opacity. B, Coronal HASTE image negative for cholesteatoma. Surgery confirmed the presence of a retraction pocket without invasion of skin into the middle ear on the right side (not shown).

and a specificity of $82 \%$, while Vercruysse et $\mathrm{al}^{16}$ reported values of $81 \%$ and $100 \%$ for primary cholesteatomas and $12.5 \%$ and $100 \%$ for residual/recurrent cholesteatomas. In EP DWI, the relatively thick sections and presence of air-bone susceptibility artifacts account for these problems. ${ }^{17}$

\section{The Role of Non-Echo-Planar Diffusion-Weighted MR Imaging in the Diagnosis of Cholesteatomas}

Non-EP DWI (including HASTE and PROPELLER sequences) has shown increased sensitivity and specificity, with fewer artifacts and thinner sections, allowing the detection of cholesteatomas measuring 2-3 mm. ${ }^{12}$ Several studies have been published since 2006 showing promising results for this technique. ${ }^{18-28}$ Recently, Li et $\mathrm{al}^{29}$ summarized these findings in a meta-analysis including 10 articles (342 patients), reporting an overall sensitivity of $94 \%$ and specificity of $94 \%$. False-negatives are rare, often caused by motion artifacts or self-evacuating "mural" cholesteatomas, and false-positives are very infrequent. ${ }^{10,12,15,30}$ Other advantages to DWI are its short acquisition times (2-5 minutes) and avoidance of intravenous contrast. ${ }^{22,31}$

One of the most studied uses for non-EP DWI in middle ear disease is as an alternative to second-look surgery in canal wall-up mastoidectomies, ${ }^{32}$ in which the posterior wall of the external ear canal is preserved. This makes postoperative care easier but impedes direct observation of the mastoidectomy during otomicroscopy, making it impossible to clinically detect residual and recurrent cholesteatomas. ${ }^{33}$

Current indications for DWI in cholesteatomas have focused on congenital and residual/recurrent disease, ${ }^{10}$ because they can be hidden or difficult to evaluate via otoscopy and postsurgical inflammation may lead to confusion on CT and standard MR imaging. DWI is also helpful in distinguishing differential diagnoses of middle ear masses, especially when combined with delayed postcontrast T1WI.

The usefulness of non-EP DWI for detecting patients with incipient disease has been less well-studied. Although excellent previous reports by Pizzini et al, ${ }^{23}$ De Foer et al, ${ }^{26}$ Profant et al, ${ }^{27}$ and Ilica et $\mathrm{al}^{34}$ have included primary cholesteatomas among their cases, our research differs in that this was a prospective study that included only retraction pockets with suspicion of cholesteatoma after evaluation by an expert otologic committee. All patients had surgical confirmation, and the data did not include other groups such as recurrent disease. Our aim was to specifically evaluate the diagnostic accuracy of non-EP DWI in difficult cases in which the presence of a middle ear cholesteatoma behind a tympanic retraction pocket was unclear.

The pathogenesis of primary acquired cholesteatomas is complicated and multifactorial, but retraction pockets seem to play an important role ${ }^{35}$; however, it remains a matter of debate as to whether this pocket can be considered a cholesteatoma. To our knowledge, there are few MR imaging-based articles in the literature assessing the presence of clinically occult cholesteatomas behind tympanic retraction pockets.

\section{Use of Non-EP DW on Tympanic Retraction Pockets}

On the basis of our results, we propose that HASTE is a highly sensitive and specific test in the evaluation of high-risk tympanic retraction pockets, where otomicroscopy alone cannot evaluate the extension of skin into the middle ear by direct observation through the external ear canal. CT showing a middle ear mass and/or bone erosion is not specific for cholesteatoma, nor is standard MR imaging. Because DWI gives poor anatomic information, does not evaluate bone erosion, and is not exempt from false-positives and negatives, all of these imaging techniques should be considered as complementary.

In agreement with the literature, we were able to detect cholesteatomas as small as $3 \mathrm{~mm}$. Furthermore, $40 \%$ of all HASTEpositive patients in our study had cholesteatomas of $\leq 5 \mathrm{~mm}$, which could have been missed if EP DWI had been used instead. $^{12,24}$

There was only 1 false-negative in an infected, "wet" cholesteatoma with otorrhea larger than $2-3 \mathrm{~mm}$. We believe that this could be explained by an alteration in the restriction pattern of the suppurated cholesteatoma, but further studies are needed to confirm or refute this hypothesis.

Although the number of subjects is limited because of the specific subset of patients included, this study might broaden the clinical applicability of non-EP DWI, especially in cases in which the diagnosis is uncertain and a more conservative approach is 
desirable (dangerous tympanic retraction on the better/only hearing ear, high anesthetic risk). Because most cases of cholesteatoma can be adequately diagnosed with otoscopy and CT, judicious use of HASTE MR imaging is important and should be considered only when clinical benefit is expected.

\section{CONCLUSIONS}

We have demonstrated that preoperative HASTE MR imaging is highly accurate with regard to detecting the presence or absence of cholesteatoma in tympanic retraction pockets. Early diagnosis of cholesteatomas arising from tympanic retraction pockets could help to better select patients requiring surgery, thus avoiding unnecessary and aggressive procedures.

Disclosures: Andrés Alvo-RELATED: Grant: Sociedad Chilena de Otorrinolaringología, ${ }^{*}$ Comments: The grant was earned among projects from members of the Chilean Society of Otorhinolaryngology, to finance relevant clinical or basic research. Cristian Garrido-RELATED: Grant: Sociedad Chilena de Otorrinolaringologia. * Carlos E. Stott—RELATED: Grant: Sociedad Chilena de Otorrinolaringologia.* Paul H. Delano—RELATED: Grant: Sociedad Chilena de Otorrinolaringologia, ${ }^{\star}$ Comments: Sociedad Chilena de Otorrinolaringologia funded MR imaging for patients recruited in this study (research grant). *Money paid to the institution.

\section{REFERENCES}

1. Semaan MT, Megerian CA. The pathophysiology of cholesteatoma. Otolaryngol Clin North Am 2006;39:1143-59

2. Nevoux J, Lenoir M, Roger G, et al. Childhood cholesteatoma. Eur Ann Otorhinolaryngol Head Neck Dis 2010;127:143-50

3. Chang P, Kim S. Cholesteatoma: diagnosing the unsafe ear. Aust Fam Physician 2008;37:631-38

4. Wu JF, Jin Z, Yang JM, et al. Extracranial and intracranial complications of otitis media: 22-year clinical experience and analysis. Acta Otolaryngol 2012;132:261-65

5. Alzahrani M, Saliba I. Tympanic membrane retraction pocket staging: is it worthwhile? Eur Arch Otorhinolaryngol 2014;271:1361-68

6. Ayache D, Darrouzet V, Dubrulle F, et al. Imaging of non-operated cholesteatoma: clinical practice guidelines. Eur Ann Otorhinolaryngol Head Neck Dis 2012;129:148-52

7. Tatlipinar A, Tuncel A, Öğredik EA, et al. The role of computed tomography scanning in chronic otitis media. Eur Arch Otorhinolaryngol 2012;269:33-38

8. Payal G, Pranjal K, Gul M, et al. Computed tomography in chronic suppurative otitis media: value in surgical planning. Indian J Otolaryngol Head Neck Surg 2012;64:225-29

9. Ayache D, Williams MT, Lejeune D, et al. Usefulness of delayed postcontrast magnetic resonance imaging in the detection of residual cholesteatoma after canal wall-up tympanoplasty. Laryngoscope 2005;115:607-10

10. Más-Estellés F, Mateos-Fernández M, Carrascosa-Bisquert B, et al. Contemporary non-echo-planar diffusion-weighted imaging of middle ear cholesteatomas. Radiographics 2012;32:1197-213

11. Jindal M, Doshi J, Srivastav M, et al. Diffusion-weighted magnetic resonance imaging in the management of cholesteatoma. Eur Arch Otorhinolaryngol 2010;267:181-85

12. De Foer B, Vercruysse JP, Spaepen M, et al. Diffusion-weighted magnetic resonance imaging of the temporal bone. Neuroradiology 2010;52:785-807

13. Ganaha A, Outa S, Kyuuna A, et al. Efficacy of diffusion-weighted magnetic resonance imaging in the diagnosis of middle ear cholesteatoma. Auris Nasus Larynx 2011;38:329-34

14. Yoshida T, Ito K, Adachi N, et al. Cholesteatoma of the petrous bone: the crucial role of diffusion-weighted MRI. Eur Arch Otorhinolaryngol 2005;262:440-41

15. Mateos-Fernández M, Mas-Estellés F, de Paula-Vernetta C, et al. The role of diffusion-weighted magnetic resonance imaging in cholesteatoma diagnosis and follow-up: study with the diffusion PROPELLER technique [in English, Spanish]. Acta Otorrinolaringol Esp 2012;63:436-42

16. Vercruysse JP, De Foer B, Pouillon M, et al. The value of diffusionweighted MR imaging in the diagnosis of primary acquired and residual cholesteatoma: a surgical verified study of 100 patients. Eur Radiol 2006;16:1461-67

17. Moura M, Taranto D, Garcia M. Cholesteatoma: utility of non-echoplanar diffusion-weighted imaging. Radiol Bras 2012;45:283-87

18. De Foer B, Vercruysse JP, Pilet B, et al. Single-shot, turbo spin-echo, diffusion-weighted imaging versus spin-echo-planar, diffusionweighted imaging in the detection of acquired middle ear cholesteatoma. AJNR Am J Neuroradiol 2006;27:1480-82

19. Dubrulle F, Souillard R, Chechin D, et al. Diffusion-weighted MR imaging sequence in the detection of postoperative recurrent cholesteatoma. Radiology 2006;238:604-10

20. Dhepnorrarat RC, Wood B, Rajan GP. Postoperative non-echo-planar diffusion-weighted magnetic resonance imaging changes after cholesteatoma surgery: implications for cholesteatoma screening. Otol Neurotol 2009;30:54-58

21. Lehmann P, Saliou G, Brochart C, et al. 3T MR imaging of postoperative recurrent middle ear cholesteatomas: value of periodically rotated overlapping parallel lines with enhanced reconstruction diffusionweighted MR imaging. AJNR Am J Neuroradiol 2009;30:423-27

22. Rajan GP, Ambett R, Wun L, et al. Preliminary outcomes of cholesteatoma screening in children using non-echo-planar diffusionweighted magnetic resonance imaging. Int J Pediatr Otorhinolaryngol 2010;74:297-301

23. Pizzini FB, Barbieri F, Beltramello A, et al. HASTE diffusion-weighted 3-Tesla magnetic resonance imaging in the diagnosis of primary and relapsing cholesteatoma. Otol Neurotol 2010;31:596-602

24. Huins CT, Singh A, Lingam RK, et al. Detecting cholesteatoma with non-echo planar (HASTE) diffusion-weighted magnetic resonance imaging. Otolaryngol Head Neck Surg 2010;143:141-46

25. Plouin-Gaudon I, Bossard D, Fuchsmann C, et al. Diffusionweighted MR imaging for evaluation of pediatric recurrent cholesteatomas. Int J Pediatr Otorhinolaryngol 2010;74:22-26

26. De Foer B, Vercruysse JP, Bernaerts A, et al. Middle ear cholesteatoma: non-echo-planar diffusion-weighted MR imaging versus delayed gadolinium-enhanced T1-weighted MR imaging-value in detection. Radiology 2010;255:866-72

27. Profant M, Sláviková K, Kabátová Z, et al. Predictive validity of MRI in detecting and following cholesteatoma. Eur Arch Otorhinolaryngol 2012;269:757-65

28. Khemani S, Lingam RK, Kalan A, et al. The value of non-echo planar HASTE diffusion-weighted MR imaging in the detection, localisation and prediction of extent of postoperative cholesteatoma. Clin Otolaryngol 2011;36:306-12

29. Li PM, Linos E, Gurgel RK, et al. Evaluating the utility of non-echoplanar diffusion-weighted imaging in the preoperative evaluation of cholesteatoma: a meta-analysis. Laryngoscope 2013;123:1247-50

30. Karandikar A, Goh J, Loke SC, et al. Mucous retention cyst of temporal bone: a mimic of cholesteatoma on DW-MRI. Am J Otolaryngol 2013;34:753-54

31. Sharifian H, Taheri E, Borghei P, et al. Diagnostic accuracy of nonecho-planar diffusion-weighted MRI versus other MRI sequences in cholesteatoma. J Med Imaging Radiat Oncol 2012;56:398-408

32. Schwartz KM, Lane JI, Bolster BD Jr, et al. The utility of diffusionweighted imaging for cholesteatoma evaluation. AJNR Am J Neuroradiol 2011;32:430-36

33. Nikolopoulos TP, Gerbesiotis P. Surgical management of cholesteatoma: the two main options and the third way-atticotomy/limited mastoidectomy. Int J Pediatr Otorhinolaryngol 2009;73:1222-27

34. Ilıca AT, Hıdır Y, Bulakbaşı N, et al. HASTE diffusion-weighted MRI for the reliable detection of cholesteatoma. Diagn Interv Radiol 2012;18:153-58

35. Louw L. Acquired cholesteatoma pathogenesis: stepwise explanations. J Laryngol Otol 2010;124:587-93 